\title{
Long-lived photoexcited states in polydiacetylenes with different molecular and supramolecular organization
}

\author{
D. Comoretto, I. Moggio, C. Cuniberti, and G. Dellepiane \\ Dipartimento di Chimica e Chimica Industriale, Università degli Studi di Genova, via Dodecaneso 31, I-16146 Genova, Italy \\ M. E. Giardini \\ Istituto Nazionale per la Fisica della Materia, Dipartimento di Fisica “A. Volta,’ Università degli Studi di Pavia, via Bassi 6, \\ I-27100 Pavia, Italy \\ A. Borghesi \\ Istituto Nazionale per la Fisica della Materia, Dipartimento di Fisica, Università degli Studi di Modena, via Campi 213/a, \\ I-41100 Modena, Italy \\ (Received 26 December 1996; revised manuscript received 10 April 1997)
}

\begin{abstract}
With the aim of determining the importance of the molecular and supramolecular organization on the excited states of polydiacetylenes, we have studied the photoinduced absorption spectra of the red form of poly[1,6bis(3,6-didodecyl- $N$-carbazolyl)-2,4-hexadiyne] (polyDCHD- $S$ ) and the results compared with those of the blue form of the same polymer. An interpretation of the data is given in terms of both the conjugation length and the interbackbone separation also in relation to the photoinduced absorption spectra of both blue and red forms of poly[1,6-bis( $N$-carbazolyl)-2,4-hexadiyne] (polyDCHD), which does not carry the alkyl substituents on the carbazolyl side groups. Information on the above properties is derived from the analysis of the absorption and Raman spectra of this class of polydiacetylenes. [S0163-1829(97)03039-7]
\end{abstract}

\section{INTRODUCTION}

The use of semiconducting conjugated polymers in different fields of application requires the preparation of novel systems that combine processability to outstanding electronic properties. Among these polymers, the polydiacetylenes (PDA's) have enjoyed a period of renaissance ${ }^{1-14}$ and the synthesis of novel PDA's has enlarged our knowledge on their properties, in particular, on the values of their nonlinear susceptibilities. ${ }^{15,16}$

In the last five years we have been interested in the study of a class of attractive PDA's, the polycarbazolyldiacetylenes, ${ }^{5,17-22}$ in particular, of poly[1,6-di ( $N$-carbazolyl)-2,4-hexadiyne] (polyDCHD), ${ }^{5,19-26}$ which is, however, insoluble. With the aim of improving its processability we have prepared a modified polyDCHD by inserting long aliphatic chains on the carbazolyl side groups. ${ }^{27}$ The novel polymer poly[1,6-bis(3,6-didodecyl$N$-carbazolyl)-2,4-hexadiyne] (polyDCHD-S) (Fig. 1) exhibits both a blue and a red form, the former unsoluble while the latter can be dissolved in the most common organic solvents. Its solubility is, however, somewhat lower than that of PDA$n \mathrm{BCMU} \quad\left[R=R^{\prime}=-\left(\mathrm{CH}_{2}\right)_{n} \mathrm{OCONHCH}_{2} \mathrm{COO}\left(\mathrm{CH}_{2}\right)_{3} \mathrm{CH}_{3}\right.$, $n=3,4] .{ }^{28}$ The two forms have been completely characterized with spectroscopic techniques.

The blue form of polyDCHD- $S$ exhibits electronic and Raman spectra very similar to those of polyDCHD (Ref. 27) but its photoinduced spectrum reveals very different features. $^{29}$ This different behavior has been interpreted in terms of different relaxation processes taking place in the two polymers and mainly related to their different interchain separations.

The red form, studied in solution, as a cast film or as a stable blend in polyethylene (PE), shows a lower conjugation length relative to the blue form as revealed by its electronic and Raman spectra here discussed. The photoinduced absorption spectrum of the red polyDCHD-S is instead quite similar to that of the blue form.

In this paper we are mainly concerned with the discussion of the photoinduced spectra of both the cast film and the PE blend of the red form of polyDCHD- $S$ in relation to those of the blue form. This comparison should shed further light on the dynamics of the long-lived photogenerated states in polydiacetylenes, also in relation to their molecular and supramolecular organization.

\section{EXPERIMENT}

Blends of red polyDCHD-S and PE have been prepared by mixing the polymer in chlorobenzene with low-density

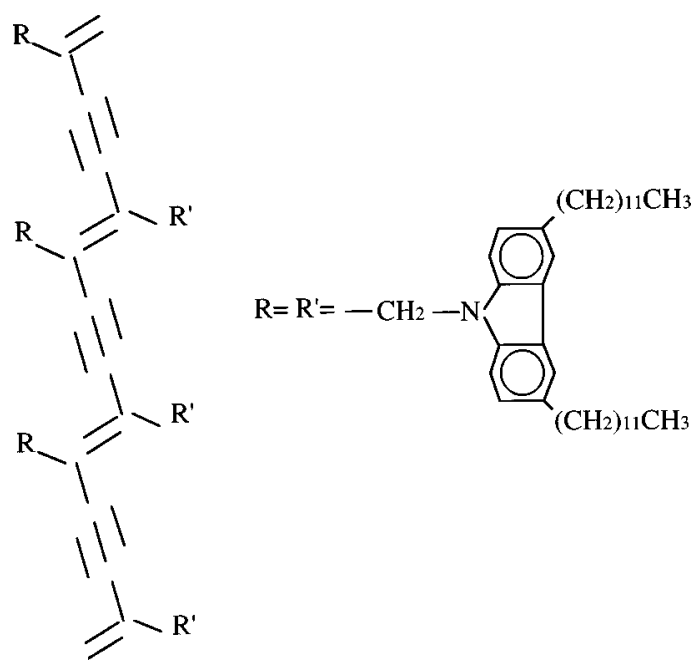

FIG. 1. Chemical structure of polyDCHD-S polymer. 
polyethylene. After heating the mixture to $120^{\circ} \mathrm{C}$, the obtained blend was cooled to room temperature. The mass sandwiched between two microscope cover glasses was transferred into a SPECAC film maker where it was again heated up to $140{ }^{\circ} \mathrm{C}$ and then cooled to room temperature.

The electronic absorption spectra have been recorded using a Cary 5E spectrophotometer operating in the range 300$2200 \mathrm{~nm}$ equipped with a liquid-nitrogen cryostat; the spectral resolution of all spectra was $\sim 1 \mathrm{~nm}$.

The Raman spectra have been recorded with a Fouriertransform Raman spectrometer Bruker FRS100 working with a Nd-YAG (yttrium aluminum garnet) laser $(1064 \mathrm{~nm})$.

The photoinduced absorption (PA) spectra have been recorded using a homemade experimental setup. A continuous wave (cw) $\mathrm{Ar}^{+}$laser, mechanically chopped, has been used as pump and a tungsten-halogen lamp as probe. The excitation wavelengths were the 488,514 , and $457 \mathrm{~nm}$ lines of the laser. The differential changes of sample transmission $(\Delta T)$ due to pump excitation were dispersed by a McPherson 218 monochromator and collected by a cooled $\mathrm{PbS}$ detector in the $0.6-1.4 \mathrm{eV}$ range and with a photomultiplier tube in the $1.4-2.3 \mathrm{eV}$ range. A Stanford SR850 DSP lock-in amplifier allowed a phase-sensitive detection. The PA spectra $(-\Delta T / T)$ have been obtained by normalizing $\Delta T$ to the transmission $(T)$ of the sample. All the low-temperature measurements have been performed with an hydrogenhelium expansion cryostat.

\section{RESULTS AND DISCUSSION}

Figure 2(a) shows the room-temperature absorption spectra of the solution, of the solution cast film, and of the $0.015 \%$ blend of red polyDCHD- $S$ and PE. In addition to the main excitonic peak $(\sim 541 \mathrm{~nm} ; \sim 2.29 \mathrm{eV})$, all the spectra exhibit a shoulder around $2.4 \mathrm{eV}$ presumably due to unresolved vibronic structures and the spectral features around $3.5 \mathrm{eV}$ originated by the transitions of the carbazolyl substituents. Unlike the case of polyarylenevinylenes, ${ }^{30,31}$ whose absorption spectra shift in the blend toward lower energy relative to cast film, in our case no appreciable variation is observed in the excitonic absorption region. However, a shift toward lower energies is observed in the carbazolyl transitions when the polymer is blended in PE, probably due to the interactions of the long aliphatic chains with the PE matrix. Moreover, it is evident that the excitonic bands of the solution are sharper and then more resolved than those of both cast film and blend. This observation is more clearly shown in Fig. 2(b) where the first derivative of the absorption spectrum is displayed. When the polymer is in the solid state, in addition to the broadening effect due to the large distribution of conjugation lengths, it is also evident the presence of a rather weak transition around $2 \mathrm{eV}$ due to a very small amount of aggregates of the blue form.

Upon lowering the temperature of the red films to $77 \mathrm{~K}$ only a very small redshift $(7 \mathrm{~nm})$ of the excitonic absorption spectrum is observed without evidence of sharpening effects, in contrast with the absorption of the blue phase where both these effects are present. ${ }^{5,22,27}$ This comparison is reported for the red blend and the blue pellet in Fig. 3. All these results indicate that the excitonic absorption spectrum of the red polyDCHD-S is practically not affected by the environ-
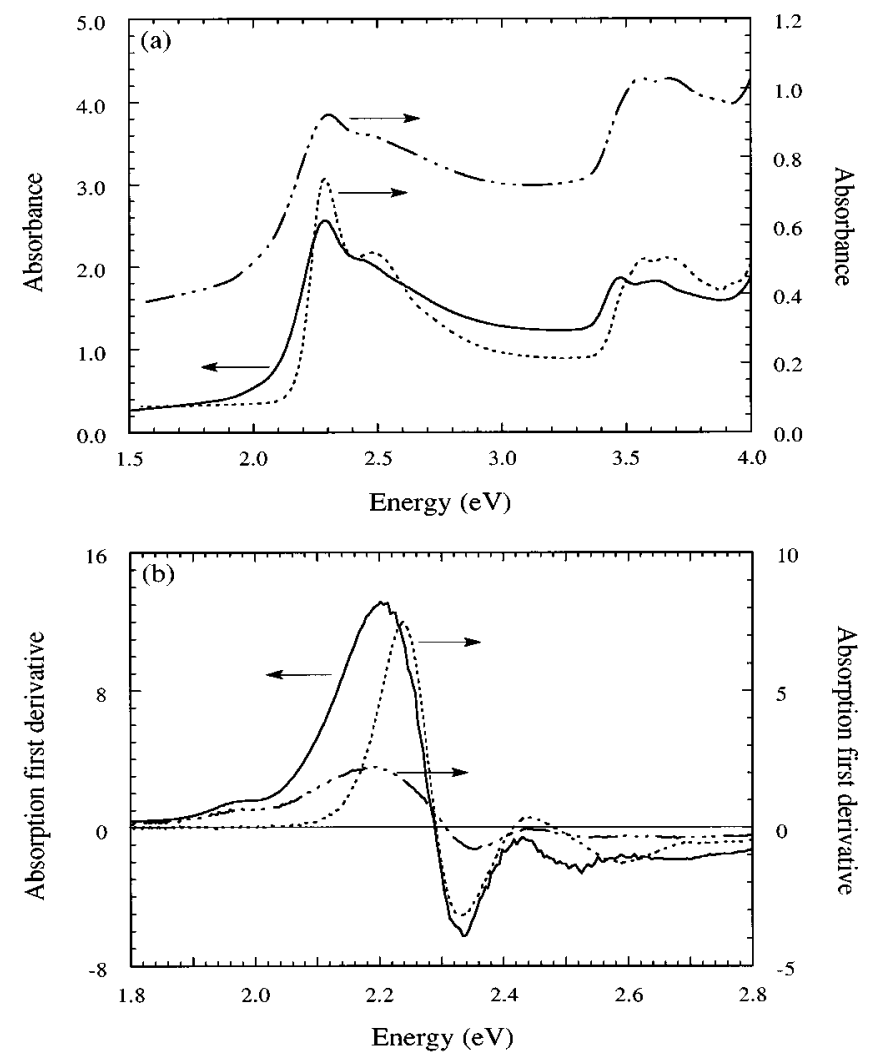

FIG. 2. (a) Room-temperature absorption spectra of the red form of polyDCHD-S: full line, blended in PE matrix; dot-dashed line, cast film; dotted line, chlorobenzene solution. (b) First derivative of the same spectra reported in (a).

ment, probably because the rigid polydiacetylenic skeleton, coupled in our case with massive side groups, inhibits the possibility of conformational variation.

When compared with the blue form in the $\mathrm{KBr}$ pellet, the spectra of the red form are indicative of a shorter effective conjugation length accompanied by a broadening of its distribution. In the inset of Fig. 3 the luminescence spectrum of the red films is reported and compared with that of the blue form. Besides some band-shape differences due to the different self-absorption in the two samples, a large Stokes shift in

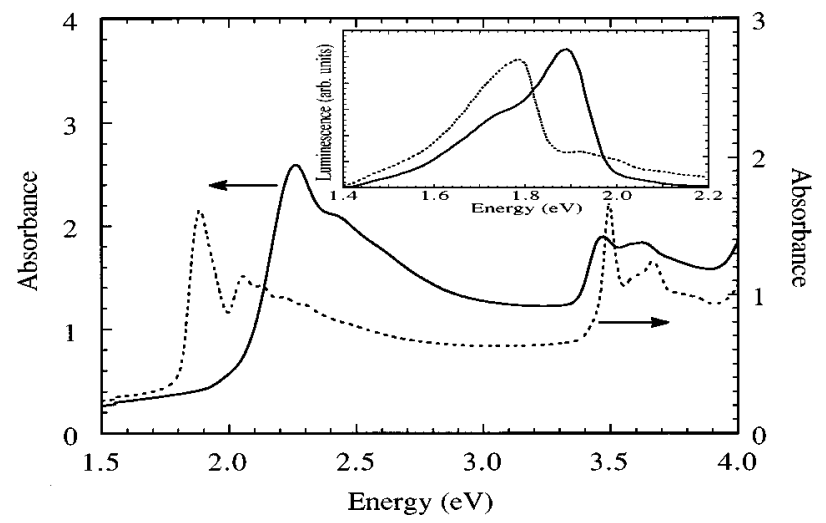

FIG. 3. Liquid-nitrogen absorption spectra of polyDCHD-S as red blend in $\mathrm{PE}$ (full line) and blue $\mathrm{KBr}$ pellet (dashed line). In the inset the luminescence spectra of the same polymers are also reported $\left(T=77 \mathrm{~K}, \lambda_{\text {exc }}=488 \mathrm{~nm}\right)$. 


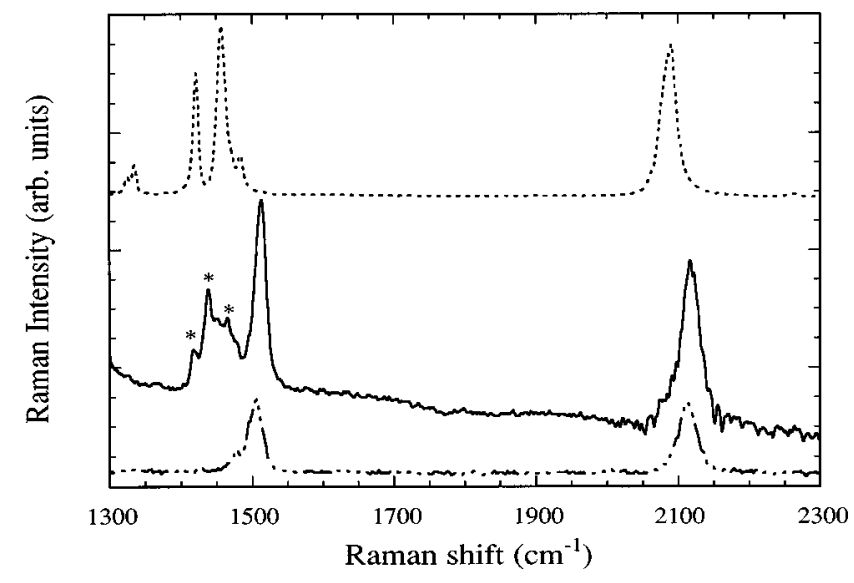

FIG. 4. Raman spectra $\left(\lambda_{\text {exc }}=1064 \mathrm{~nm}\right)$ of polyDCHD- $S$ as red blend in PE (full line), red cast film (dot-dashed line), and blue $\mathrm{KBr}$ pellet (dashed line). The stars indicate the Raman bands of the PE matrix.

the red form relative to the blue one is observed. This fact is attributed to a relaxation before emission towards states that are more displaced with respect to the singlet excitonic level.

The reduction of the effective conjugation length of the red form is also confirmed by the values of the Raman frequencies, which are at higher wave numbers with respect to those of the blue form. Indeed, as shown in Fig. 4, the Raman lines assigned to the $\mathrm{C} \equiv \mathrm{C}$ and $\mathrm{C}=\mathrm{C}$ stretchings of the blue form appear at 2091 and $1457 \mathrm{~cm}^{-1}$, respectively (note the multiline structure in the double bond region previously assigned to a Fermi resonance $e^{27,32,33}$ ). The Raman lines of the same vibrational modes in the red form appear at 2112 and $1504 \mathrm{~cm}^{-1}$ (cast film) and at 2117 and $1513 \mathrm{~cm}^{-1}$ (blend). Note the complex structure around $1450 \mathrm{~cm}^{-1}$ due to the vibrations of the PE matrix. The low concentration of the red solution does not allow to evidence the Raman spectrum out of resonance. Preliminary measurements in resonance ${ }^{34}$ show Raman shifts at 2118 and $1515 \mathrm{~cm}^{-1}$, that is, without appreciable variation with respect to those observed in the solid state.

The PA spectrum of the red blend at $77 \mathrm{~K}$, obtained with the 488-nm excitation line, is reported for both the in-phase and out-phase detections in Figs. 5(a) and 5(b), respectively, and there compared with the PA spectrum of the blue form measured under the same experimental conditions. ${ }^{29}$ The PA spectrum of the red cast film (not reported here) is practically identical to that of the blend. Notice first of all that only a PA signal of considerable intensity $\left(-\Delta T / T \sim 3 \times 10^{-2}\right)$ is observed at $1.4 \mathrm{eV}$. This band appears at about the same energy of the PA peak of the blue form but its shape is more asymmetrical, with no evident shoulder in its low-energy side. In its out-of-phase detection it reveals a decrease of intensity similar to that experienced by the blue form. But, unlike the latter case, no signals due to long-lived charged excitations could be detected here. ${ }^{35}$

The spectral region where the bleaching occurs shows very different features for the two forms. For the red form, the bleaching is observed in both the in-phase and out-ofphase detections while for the blue form it becomes relevant only in the out-of-phase spectrum. In addition, in this latter case, the bleaching effect is much stronger than the PA signal. The contribution of the luminescence emission does not

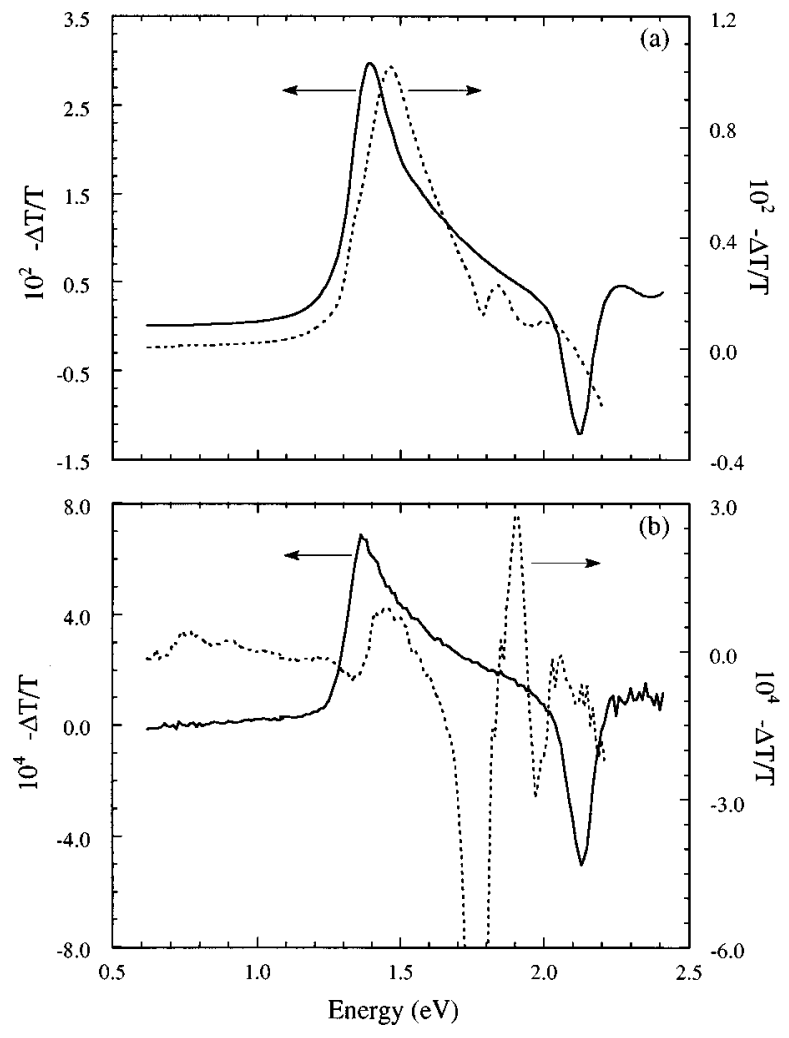

FIG. 5. Liquid-nitrogen photoinduced absorption spectra of polyDCHD-S both as red blend in PE (full line) and blue KBr pellet (dashed line) for in-phase (a) and out-of-phase (b) detections $\left(T=77 \mathrm{~K}, \lambda_{\text {exc }}=488 \mathrm{~nm}\right.$, modulation frequency $\left.20 \mathrm{~Hz}\right)$.

seem large enough to justify these different data. Notice also the lack of oscillations in the bleaching region of the red blend, which could be related with the absence of photoexcited charged states in this polymer. The same behavior has been previously observed in other conjugated systems. ${ }^{19,36-38}$ Unfortunately we cannot discuss in detail the relation between photoexcited charged states and oscillations in the bleaching region because no electromodulated spectra of our polymers are so far reported. The PA spectra of the blend obtained with both the 457- and 514-nm exciting lines show features identical to those obtained with the 488 -nm excitation.

These results suggest that for both the red blend and cast film the formation of charged states is inhibited. This fact could be explained in the case of the red blend in terms of quenching of the interchain decay routes due to dilution of the polymer in the PE matrix. However, the lack of signals associated with charged excitations also in the red cast film suggests the existence of another mechanism responsible for the decrease of the interchain coupling essential for their formation. In fact, charged photoexcited states can be created only if an electron is transferred from one chain to another one. It is well known indeed that this process, mediated by the interchain coupling, is strictly related to the overlap of the $p_{z}$ orbitals of adjacent chains. Because the chain conformation of PDA's in the red phase is somewhat twisted from the planar conformation typical of the blue form (presumably due to the nonperfect alignment of the side groups that reduces the long-range interchain ordering ${ }^{39,40}$ ), only a relative 

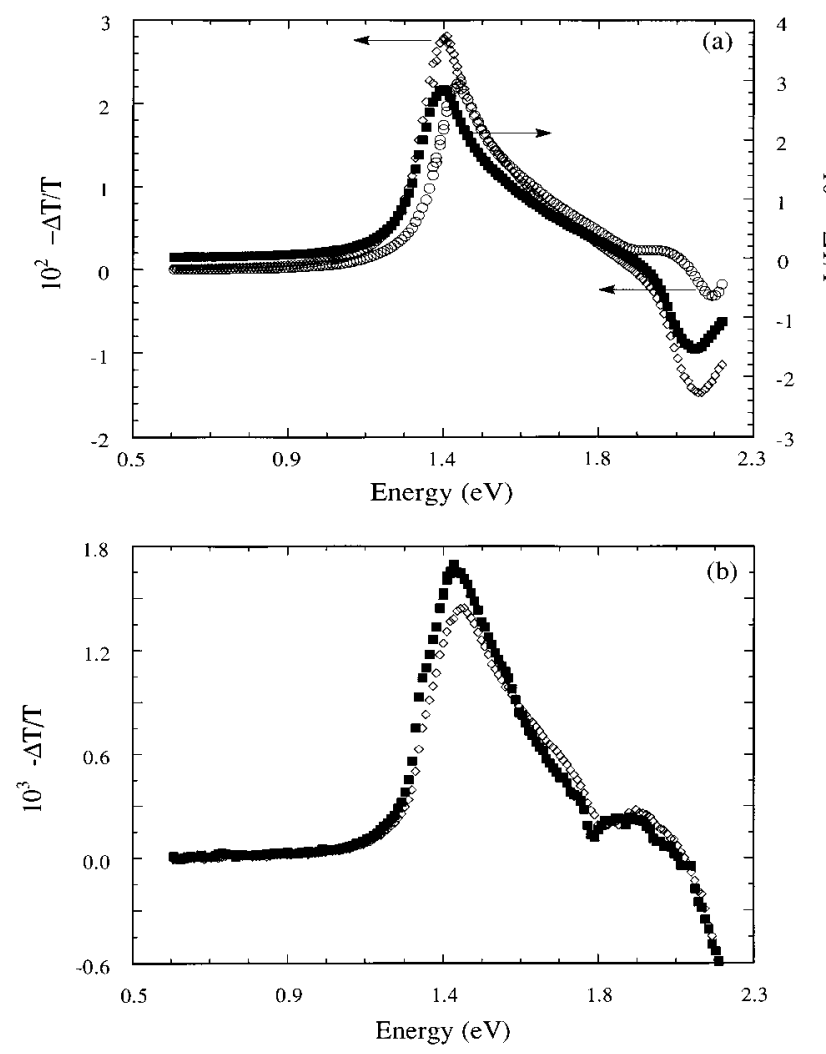

FIG. 6. In-phase photoinduced absorption spectra of (a) polyDCHD- $S$ red blend in PE. Full squares, $T=20 \mathrm{~K}$ and $\lambda_{\text {exc }}$ $=488 \mathrm{~nm}$; open diamonds, $T=77 \mathrm{~K}$ and $\lambda_{\text {exc }}=488 \mathrm{~nm}$; open circles, $T=300 \mathrm{~K}$ and $\lambda_{\mathrm{exc}}=514 \mathrm{~nm}$, modulation frequency $20 \mathrm{~Hz}$; (b) polyDCHD- $S$ blue form. Open diamonds, $T=77 \mathrm{~K}$ and $\lambda_{\text {exc }}$ $=488 \mathrm{~nm}$; full squares, $T=20 \mathrm{~K}$ and $\lambda_{\mathrm{exc}}=488 \mathrm{~nm}$.

small overlap develops between the backbone orbitals of different chains.

In order to determine the nature of the nonlinear excitations responsible for the PA spectrum, we have studied the dependence of the 1.4-eV signal on the temperature, laser power, and modulation frequency.

Practically no changes of the intensity of the PA spectrum with the temperature have been observed in the $20-300 \mathrm{~K}$ range. This result, illustrated in Fig. 6(a), indicates that deeptrapped long-lived nonlinear excitations are associated with the 1.4-eV signal. The slight blueshift of the peak position at $300 \mathrm{~K}$ is comparable with the shift of the excitonic absorption reported above. Note that also the bleaching exhibits the same shift. A similar temperature dependence has been observed also for the 1.45 PA peak of the blue form of the same polymer as shown in Fig. 6(b). Further information on the nature of these quasiparticles has been obtained by measuring the dependence of the PA band on the chopper frequency. The results obtained for two different laser powers and for $T=77 \mathrm{~K}$ are reported in Figs. 7(a) and 7(b) for the red and blue forms of polyDCHD-S, respectively. In both cases the band intensity corresponding to different laser powers is independent on the chopper frequency up to 200 $\mathrm{Hz}$ and then begins to roll off for higher frequencies. The fact that for both forms the knee position is practically independent on the laser power is unambiguously indicative of a monomolecular decay kinetics described by the equation ${ }^{19}$
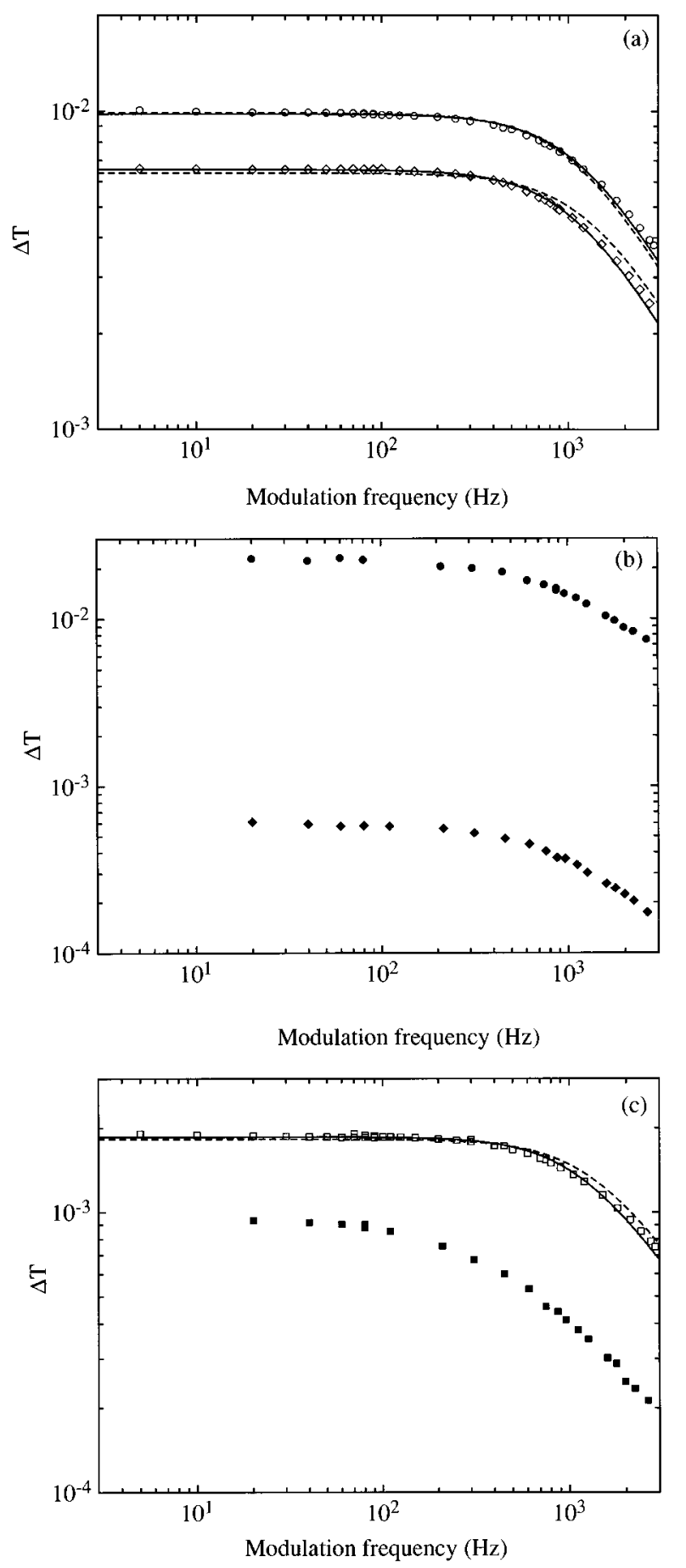

FIG. 7. Modulation frequency dependence of the photoinduced absorption peak in (a) red polyDCHD- $S$ blend in PE. $T=77 \mathrm{~K}$, $\lambda_{\text {exc }}=488 \mathrm{~nm}, I=70 \mathrm{~mW}$ (open diamonds), and $I=140 \mathrm{~mW}$ (open circles). (b) Blue polyDCHD- $S, T=77 \mathrm{~K}, \lambda_{\text {exc }}=488 \mathrm{~nm}$; full diamonds, $I=70 \mathrm{~mW}$; full circles, $I=200 \mathrm{~mW}$. (c) $T=20 \mathrm{~K}, \lambda_{\mathrm{exc}}=488$ $\mathrm{nm}$; open squares, red polyDCHD- $S$ blend in PE $(I=100 \mathrm{~mW})$; full squares, blue polyDCHD-S $(I=200 \mathrm{~mW})$. Full and dashed lines are fittings with monomolecular kinetic law (see text).

$$
\frac{d n}{d t}=G(t)-\frac{n}{\tau}
$$

Here $d n / d t$ is the change in the photoexcitations density per unit time, $\tau$ is the lifetime of the carrier, and $G(t)$ is the 
pumping term with angular frequency $\omega$, which is usually modeled as $g I[1+\cos (\omega t)] . I$ is the pump intensity and $g$ the efficiency of the photoexcitations generation. This equation has been solved and its solution for the steady-state case is given by

$$
\begin{gathered}
n(t)=g I \tau\left[\frac{1}{\sqrt{1+\omega^{2} \tau^{2}}} \cos (\omega t-\phi)+1\right], \\
\tan (\phi)=\omega \tau,
\end{gathered}
$$

where $\phi$ is the phase of the outcoming radiation. From this equation, the amplitude of the photoexcitation density that is proportional to $\Delta T$ can be easily derived:

$$
\Delta T \approx \frac{g I \tau}{\sqrt{1+\omega^{2} \tau^{2}}}
$$

This well-known equation predicts an $I$ dependence of the PA signal. Moreover, for $\omega \tau \gg 1$ a $\omega^{-1}$ law is predicted, while for $\omega \tau \ll 1$ the signal is independent on the chopper frequency. Note also that $\phi$ approaches 0 for very small values of the lifetime and $\phi$ approaches $\pi / 2$ for very large values of $\tau$. The in-phase $(\phi=0)$ and out-of-phase $(\phi=\pi / 2)$ photoinduced spectra can then be used to detect states with different lifetimes.

By least-square fitting of the experimental data at $77 \mathrm{~K}$ with Eq. (4) the lifetimes of the nonlinear excitations turn out to be practically identical for different laser powers either for the red $(\tau=150 \mu$ s for $70 \mathrm{~mW}$ and $\tau=145 \mu$ s for $140 \mathrm{~mW})$ and for the blue form $(\tau=210 \mu \mathrm{s}$ for $70 \mathrm{~mW}$ and $\tau=195 \mu \mathrm{s}$ for $200 \mathrm{~mW}$ ). On the other hand, lifetime ratios of $\sqrt{2}$ and $\sqrt{3}$ are predicted for a bimolecular recombination kinetics since in this case the lifetime should vary as the inverse square root of the laser intensity. ${ }^{17,19}$ At $20 \mathrm{~K}$, the lifetime for either the red $(135 \mu \mathrm{s})$ and the blue $(320 \mu \mathrm{s})$ forms turns out to be very similar to those at $77 \mathrm{~K}$, giving further support to the deep trapping of the nonlinear excitations in this polymer [Fig. 7(c)].

An additional proof of the monomolecular decay of the excitations for the red form of polyDCHD- $S$ is provided by the experimental data on the signal phase $\Phi$ (relative to that of the pumping laser), which show no variation with the laser power [Fig. 8(a)]. In fact, in a bimolecular decay process the signal phase, being related to the lifetime, should depend on the laser intensity. A further test of the monomolecular behavior of the decay process is provided by the linear dependence exhibited by the tangent of the signal phase, $\Phi$ (accurately corrected for instrumental dephasing) versus the modulation frequency. The plots based on Eq. (3) (Refs. 19 and 41) are reported in Fig. 8(b) for the same experimental conditions of Figs. 7(a) and 7(c). The values so obtained, $\tau=125 \mu \mathrm{s}$ at $70 \mathrm{~mW} / 77 \mathrm{~K}, \tau=155 \mu \mathrm{s}$ at $140 \mathrm{~mW} / 77 \mathrm{~K}$, and $\tau=115 \mu \mathrm{s}$ at $100 \mathrm{~mW} / 20 \mathrm{~K}$, are quite similar to those derived from the dependence of $\Delta T$ on the modulation frequency. A calculation of $\Delta T$ versus modulation frequency with the monomolecular law using these lifetimes is also reported in Figs. 7(a) and 7(c). Unfortunately, this kind of measurement was not carried out for the blue form previously studied. ${ }^{29}$
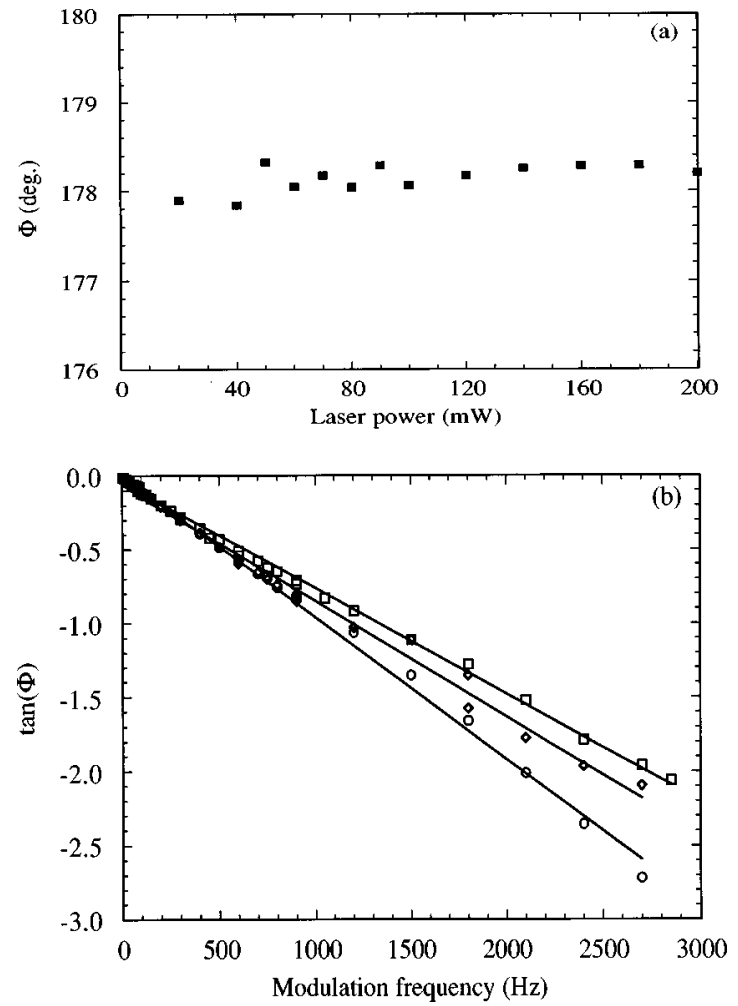

FIG. 8. Intensity dependence of the 1.4-eV photoinduced signal phase at $T=77 \mathrm{~K}, \lambda_{\text {exc }}=488 \mathrm{~nm}$, modulation frequency $20 \mathrm{~Hz}$. (b) Frequency dependence of the phase of the photoinduced signal. Open circles, $T=77 \mathrm{~K}, \lambda_{\text {exc }}=488 \mathrm{~nm}, I=140 \mathrm{~mW}$; open diamonds, $T=77 \mathrm{~K}, \lambda_{\text {exc }}=488 \mathrm{~nm}, I=70 \mathrm{~mW}$; open squares, $T=20 \mathrm{~K}$, $\lambda_{\text {exc }}=488 \mathrm{~nm}, I=100 \mathrm{~mW}$. Full lines are fittings with monomolecular decay law.

In disagreement with the monomolecular decay kinetics inferred from these data, the PA signal does not follow the linear laser intensity $(I)$ dependence expected in this case. The experimental data could in fact be fitted only with a $I^{0.73}$ law. In contrast with previous results on other PDA's, ${ }^{19}$ no linear relationship could here be found even taking into account only the measurements carried out in the low laser intensity range. This sublinear intensity dependence has been detected also for other polymers whose $\Delta T$ versus $\omega$ dependence was fitted by a monomolecular decay law. ${ }^{29,42,43} \mathrm{~A}$ possible explanation for this discrepancy may be given in terms of the saturation of either the excited-state populations as proposed for a polydiacetylene ${ }^{44}$ or of the sites available for the trapping of the photogenerated excitations. ${ }^{45,46}$

We would like to stress at this point the similarity in the behavior of the 1.4-eV signal of the red form and the $1.45-\mathrm{eV}$ peak of the blue form ${ }^{29}$ upon changing the parameters associated with the PA experiments. For this reason it seems reasonable to assign the $1.4-\mathrm{eV}$ signal of the red form to a triplet-to-triplet transition, as already proposed for the $1.45-\mathrm{eV}$ peak of the blue form. ${ }^{29}$ The fact that the energy of the triplet-triplet transition does not vary in the two forms of polyDCHD- $S$ is puzzling since they present different conjugation lengths as previously discussed in the present paper. On the other hand, in other systems, the energy position of the photoinduced absorption spectra has been shown to depend on their conjugation length. In particular, the PA energy of a series of well-characterized oligomers of thiophene 
depends on the inverse of their length. ${ }^{41}$ This result has been explained in terms of the different extension of the photoexcited triplet state. Also, in PDA-4BCMU, the PA band of the red form appears at lower energy with respect to the corresponding peak of the yellow form. ${ }^{47}$ An analogous behavior has been found also in the PA band of the blue and red forms of polyDCHD, where the $1.27-\mathrm{eV}$ PA peak of the blue form ${ }^{19}$ is attributed to a shallow-trapped triplet exciton with a lifetime of $230 \mu$ s at $20 \mathrm{~K}$ and much less at $77 \mathrm{~K}$ while the $1.5-\mathrm{eV}$ peak of the red form ${ }^{21}$ is assigned to a deep-trapped triplet with a very long lifetime.

The similarity of the PA data here presented for the blue and the red forms of polyDCHD-S clearly indicates that the conjugation length is not the unique parameter that determines the spectral features and properties of the PA spectra. The main difference between the PA spectra of polyDCHD and polyDCHD- $S$ is that in the former the blue form can accommodate an almost-free triplet photoexcitation, while in the latter the blue form (although with the same effective conjugation length) gives rise to a trapped exciton. The triplet excitons of the red forms are trapped for both polymers. The cause for the different behavior of the blue form of the two polymers is most likely related to the supramolecular organization that controls the extension of the triplet excitons, their dynamics, and their interactions. The interbackbone separation expected on the basis of the chemical structure of polyDCHD-S with fully extended alkyl substituents $(30 \AA)$ is also substantiated by preliminary x-ray-diffraction measurements carried out on polyDCHD- $S$ microcrystalline powder in the blue form. The more intense diffraction peak corresponds to a value for the $c$ axis of $29.5 \AA{ }^{48}$ much larger than that for polyDCHD $(17 \AA) .{ }^{49}$

The photoexcited triplets observed in the time-scale region of the PA experiments are most likely trapped on the conformational defects. The depth of the traps depends on the difference between the configurational structure of the defect and the structure of the regular chain. The different depths of the traps in the two polymers could then be related to more relevant changes required to accommodate the photoexcitation in the backbone of the polymer that exhibits larger substituents. ${ }^{50,51}$

In the case of polyDCHD-S, the lack of the interbackbone decay routes forces the triplets to be deep trapped, while in blue polyDCHD, the possibility of interchain hopping ${ }^{52}$ allow the photoexcitations to decay with intrinsic routes and then to behave as almost free excitons before being trapped. This interpretation accounts also for the very weak signal observed in this polymer at $77 \mathrm{~K}$ and for the peak enhancement at $20 \mathrm{~K}$. We would like to note here again that the absence of charged photoexcitations in the red form of polyDCHD- $S$ as well as in the red form of other PDA's (Ref. 43) may be ascribed to a different mechanism based on the loss of long-range order. The chain disorder reduces the overlap of the wave functions of different chains and inhibits the formation of charged states.

Very different is instead the situation for the yellow phase of PDA's in solution where the conjugation length is the main mechanism governing the properties of the PA spectra. In fact, this form has a strongly reduced (6-8 repeat units) (Refs. 39, 51, and 53) conjugation length relative to the red form and its PA spectrum is correspondingly high-energy shifted.

We believe that the present interpretation of the photoexcitations in polycarbazolyldiacetylenes in terms of both conjugation length and interchain coupling should be extended to other novel properly designed PDA's. Also, theoretical models including these effects could be of great help in this analysis.

We would like to stress at this point that the presence of different photoexcited triplet states due to interactions among molecules is not a novelty in conjugated systems. Absorption-detected magnetic-resonance measurements in $\mathrm{C}_{60}$ (Refs. 54 and 55) show the presence of two kinds of triplets states assigned to molecular and aggregated fullerenes.

PolyDCHD-S samples, in particular, in the high-opticalquality red form blended in PE, seem to be appealing for nonlinear optics. In fact, the charged deexcitation routes are completely suppressed relative to the neutral ones, which are responsible for the nonlinear optical response. ${ }^{56-58}$ In polyDCHD a very high value of $\chi^{(3)}$ was observed along with charged photogenerated states; in polyDCHD- $S$ the lack of charged photoexcited states suggests that an even larger nonlinear optical response could be observed.

\section{ACKNOWLEDGMENTS}

We acknowledge financial support by the Italian Ministry of University and of Scientific and Technological Research (MURST), and by the National Research Council (C.N.R.), Italy.
${ }^{1}$ W. A. Pender, A. J. Boyle, P. Lambkik, W. J. Blau, K. Mazaheri, D. J. Westland, V. Skarda, and M. Sparpaglione, Appl. Phys. Lett. 66, 786 (1995).

${ }^{2}$ M. S. Paley, D. O. Frazier, H. Abdeldeyem, S. Armstrong, and S. P. McManus, J. Am. Chem. Soc. 117, 4775 (1995).

${ }^{3}$ J. Y. Bigot, T. A. Pham, and A. Daunois, Photonics Science News 2, 6 (1996).

${ }^{4}$ T. A. Pham, A. Daunois, J.-C. Merle, J. Le Moigne, and J. Y. Bigot, Phys. Rev. Lett. 74, 904 (1995).

${ }^{5}$ D. Comoretto, G. Dellepiane, C. Cuniberti, L. Rossi, A. Borghesi, and J. Le Moigne, Phys. Rev. B 53, 15653 (1996). See also references therein.
${ }^{6}$ C. E. Masse, K. Vander Wiede, W. H. Kim, X. L. Jiang, J. Kumar, and S. Triphaty, Chem. Mater. 7, 904 (1995).

${ }^{7}$ P. T. Hammond and M. F. Rubner, Macromolecules 28, 795 (1995).

${ }^{8}$ Y. Ueda, T. Kuriyama, T. Hari, M. Watanabe, J. Ni, Y. Hattori, N. Uenishi, and T. Uemiya, Jpn. J. Appl. Phys. 34, 3876 (1995).

${ }^{9}$ J. J. Kane, R.-F. Liao, J. W. Laucher, and F. W. Fowler, J. Am. Chem. Soc. 117, 12003 (1995).

${ }^{10}$ S. A. Hambir, G. J. Blanchard, and G. L. Baker, J. Chem. Phys. 102, 2295 (1995).

${ }^{11}$ A. Berman, D. J. Ahm, A. Lio, M. Salmeron, A. Reichert, and D. Charych, Science 269, 515 (1995). 
${ }^{12}$ J. Even, B. Toudic, M. Bertault, H. Cailleau, and F. Moussa, Phys. Rev. B 52, 7142 (1995).

${ }^{13}$ Y. Hattori, M. Kubata, T. Uemiya, and G. Tanaka, Jpn. J. Appl. Phys. 34, 3871 (1995).

${ }^{14}$ W. S. Price, N. Kikuchi, H. Matsuda, K. Hayamizu, S. Okada, and H. Nakanishi, Macromolecules 28, 5363 (1995).

${ }^{15}$ M. Nisoli, V. Pruneri, V. Magni, S. De Silvestri, G. Dellepiane, D. Comoretto, C. Cuniberti, and J. Le Moigne, Appl. Phys. Lett. 65, 590 (1994)

${ }^{16}$ S. Molyneaux, H. Matsuda, A. K. Kar, B. S. Wherret, S. Okoda, and H. Nakanishi, Nonlinear Opt. 4, 299 (1993).

${ }^{17}$ G. Dellepiane, C. Cuniberti, D. Comoretto, G. Lanzani, G. F. Musso, P. Piaggio, R. Tubino, A. Borghesi, C. Dell'Erba, G. Garbarino, and L. Moramarco, Phys. Rev. B 45, 6802 (1992).

${ }^{18}$ G. Dellepiane, C. Cuniberti, P. Piaggio, G. F. Musso, D. Comoretto, G. Lanzani, A. Piaggi, and A. Borghesi, Synth. Met. 51, 239 (1992).

${ }^{19}$ G. Dellepiane, C. Cuniberti, D. Comoretto, G. F. Musso, G. Figari, A. Piaggi, and A. Borghesi, Phys. Rev. B 48, 7850 (1993).

${ }^{20}$ D. Comoretto, C. Cuniberti, G. F. Musso, G. Dellepiane, F. Speroni, C. Botta, and S. Luzzati, Phys. Rev. B 49, 8059 (1994).

${ }^{21}$ G. Dellepiane, D. Comoretto, C. Cuniberti, G. F. Musso, A. Piaggi, F. Speroni, C. Botta, and S. Luzzati, Synth. Met. 68, 33 (1994).

${ }^{22}$ D. Comoretto, G. Dellepiane, C. Cuniberti, G. F. Musso, L. Rossi, A. Borghesi, and J. Le Moigne, Synth. Met. 76, 27 (1996)

${ }^{23}$ T. A. Pham, A. Daunois. J.-C. Merle, J. Le Moigne, and J.-Y. Bigot, Phys. Rev. Lett. 74, 904 (1995).

${ }^{24}$ J. Le Moigne, F. Kajzar, and A. Thierry, Macromolecules 24, 2622 (1991).

${ }^{25}$ C. Jundt, G. Klein, and J. Le Moigne, Chem. Phys. Lett. 203, 37 (1993).

${ }^{26}$ S. A. Hambir, T. Yang, G. J. Blanchard, and G. L. Baker, Chem. Phys. Lett. 201, 521 (1993).

${ }^{27}$ C. Colombi, D. Comoretto, C. Cuniberti, G. F. Musso, P. Piaggio, and G. Dellepiane, Chem. Phys. 197, 1241 (1996).

${ }^{28}$ G. N. Patel, J. Polym. Sci. Polym. Lett. Ed. 16, 607 (1978).

${ }^{29}$ D. Comoretto, I. Moggio, C. Dell'Erba, C. Cuniberti, G. F. Musso, G. Dellepiane, L. Rossi, M. E. Giardini, and A. Borghesi, Phys. Rev. B 54, 16357 (1996). Note that in Fig. 4 a misprint occurred. The $P=70 \mathrm{~mW}$ curves with $T=20$ and $T=77 \mathrm{~K}$ have been inverted.

${ }^{30}$ T. W. Hagler, K. Pakbaz, K. F. Voss, and A. J. Heeger, Phys. Rev. B 44, 8652 (1991).

${ }^{31}$ S. Luzzati, P. Elmino, and A. Bolognesi, Synth. Met. 76, 23 (1996).

${ }^{32}$ S. Hankin and D. J. Sandman, Polym. Commun. 31, 22 (1990).
${ }^{33}$ S. Hankin, D. J. Sandman, E. A. Yost, and T. J. Stark, Synth. Met. 49, 281 (1992).

${ }^{34} \mathrm{~S}$. Luzzati (private communication).

${ }^{35}$ The nature of the charge photoexcited states in the spectral range $0.8-1 \mathrm{eV}$ found in the blue form is discussed in Ref. 29.

${ }^{36}$ R. A. J. Janssen, M. P. T. Christiaans, K. Pakbaz, D. Moses, J. C. Hummelen, and N. S. Sariciftci, J. Chem. Phys. 102, 2628 (1995).

${ }^{37}$ Z. V. Vardeny, J. M. Leng, and S. Frolov, Synth. Met. 69, 407 (1995).

${ }^{38}$ D. D. C. Bradley and R. H. Friend, J. Mol. Electron. 5, 19 (1989).

${ }^{39}$ A. J. Campbell and C. K. L. Davies, Polymer 36, 675 (1995).

${ }^{40}$ Z. Iqbal, N. S. Murthy, Y. P. Khanna, J. S. Szobota, R. A. Dalterio, and F. J. Owens, J. Phys. C 20, 4283 (1987).

${ }^{41}$ R. A. J. Janssen, L. Smilowitz, N. S. Sariciftci, and D. Moses, J. Chem. Phys. 101, 1787 (1994).

${ }^{42}$ R. A. J. Janssen, M. P. T. Christiaans, C. Hare, N. Martin, N. S. Sariciftci, A. J. Heeger, and F. Wudl, J. Chem. Phys. 103, 8840 (1995).

${ }^{43}$ N. S. Sariciftci, B. Kraabel, C. H. Lee, K. Pakbaz, A. J. Heeger, and D. J. Sandman, Phys. Rev. B 50, 12044 (1994).

${ }^{44}$ B. J. Greene, J. Orenstein, R. R. Millard, and L. R. Williams, Phys. Rev. Lett. 58, 2750 (1987).

${ }^{45}$ P. A. Lane, X. Wei, Z. V. Vardeny, J. Poplawski, E. Ehrenfreund, M. Ibrahim, and A. J. Frank, Synth. Met. 76, 57 (1996).

${ }^{46}$ S. Schmitt-Rink, D. C. Chemla, and D. A. B. Miller, Adv. Phys. 38, 89 (1989).

${ }^{47}$ R. H. Austin, G. L. Baker, S. Etemad, and R. Thompson, J. Chem. Phys. 90, 6642 (1989).

${ }^{48} \mathrm{~W}$. Porzio (private communication).

${ }^{49}$ P. A. Apgar and K. C. Lee, Acta Crystallogr. Sect. B 34, 957 (1978).

${ }^{50}$ V. Dobrosavljevic and R. M. Stratt, Phys. Rev. B 35, 2781 (1987).

${ }^{51}$ A. J. Campbell and C. K. L. Davies, Polymer 35, 4787 (1994).

${ }^{52}$ S. Abe, Synth. Met. 85, 1015 (1997).

${ }^{53}$ G. Wenz, M. A. Müller, M. Schmidt, and G. Wegner, Macromolecules 17, 837 (1984).

${ }^{54}$ A. Angerhofer, J. U. von Schütz, D. Widmann,W. H. Müller, H. U. ter Meer, and H. Sixl, Chem. Phys. Lett. 217, 403 (1994).

${ }^{55}$ X. Wei and Z. V. Vardeny, Phys. Rev. B 52, R2317 (1995).

${ }^{56}$ B. I. Green, J. Orenstein, and S. Schmitt-Rink, Science 247, 679 (1990).

${ }^{57}$ S. N. Dixit, D. Guo, and S. Mazumdar, Phys. Rev. B 43, 6781 (1991).

${ }^{58}$ T. W. Hagler and A. J. Heeger, Chem. Phys. Lett. 189, 333 (1992). 